\title{
Neutron spectrometry with scintillating bolometers of LiF and sapphire
}

\author{
Noël Coron, Clara Cuesta, Eduardo García, Carlos Ginestra, Johann Gironnet, Pierre de Marcillac, \\ María Martínez, Ysrael Ortigoza, Alfonso Ortiz de Solórzano, Jorge Puimedón, Thierry Redon, \\ Tomás Rolón, María L. Sarsa, Lidia Torres, and José A. Villar
}

\begin{abstract}
Two scintillating bolometers of $\mathrm{LiF}(33 \mathrm{~g})$ and $\mathrm{Al}_{2} \mathrm{O}_{3}$ $(50 \mathrm{~g})$ at $20 \mathrm{mK}$, inside a lead shielding at the Canfranc Underground Laboratory, were irradiated with neutrons from a source of ${ }^{252} \mathrm{Cf}$. The analysis of nuclear recoils registered by sapphire and $(\mathrm{n}, \alpha)$ captures by ${ }^{6} \mathrm{Li}$ shows the feasibility of these cryogenic devices to measure the spectral flux of a neutron field. Data unfolding was done assuming that the spectral flux is a piecewise constant function defined on six energy groups. It can be solved by using non-negative least squares without additional assumptions on the neutron flux. The model provides consistent results with the spectra of the observed events in bolometers, giving a fast neutron flux of $\Phi(E>0.1 \mathrm{MeV})=0.20 \mathrm{n} \mathrm{s}^{-1} \mathrm{~cm}^{-2}$ with a $15 \%$ uncertainty after 3 hours of live time. After our analysis, it can be concluded that nuclear recoils in sapphire are
\end{abstract}

This paragraph of the first footnote will contain the date on which you submitted your paper for review.

This work was supported by the Spanish Ministerio de Economía y Competitividad, the European Regional Development Fund (MINECOFEDER) under grants FPA2008-03228, FPA2011-23749, the ConsoliderIngenio 2010 Programme under grant MULTIDARK CSD2009-00064, Ministerio de Educación y Ciencia. Programa Nacional de Movilidad de Recursos Humanos del Plan nacional de I+D+I 2008-2011, Fundación Carolina, the French CNRS/INSU (MANOLIA and BOLERO projects) and the EU project ILIAS (RII3-CT-2004-506222).

N. Coron, P. de Marcillac, T. Redon, and L. Torres are with Institut d'Astrophysique Spatiale (IAS). Bâtiment 121, Université Paris-Sud and CNRS (UMR 8617), 91405 Orsay Cedex, France (e-mail: noel.coron@ias.upsud.fr, thierry.redon@ias.u-psud.fr, lidia.torres.research@gmail.com). P. de Marcillac has recently moved to CSNSM, Batiment. 108, CNRS and Université Paris-Sud, 91405 Orsay Cedex, France (e-mail: marcillac@csnsm.in2p3.fr).

C. Cuesta was with Laboratorio de Física Nuclear y Astropartículas, Universidad de Zaragoza, 50009 Zaragoza, Spain and with Laboratorio Subterráneo de Canfranc, 22880 Canfranc Estación, Huesca, Spain. She is now with Center for Experimental Nuclear Physics and Astrophysics, University of Washington, Seattle, WA, USA (e-mail:ccuesta@uw.edu).

E. García, C. Ginestra, M. Martínez, Y. Ortigoza, A. Ortiz de Solórzano, J. Puimedón, M. L. Sarsa, J. A. Villar are with Laboratorio de Física Nuclear y Astropartículas, Universidad de Zaragoza, 50009 Zaragoza, Spain. They are also with Laboratorio Subterráneo de Canfranc, 22880 Canfranc Estación, Huesca, Spain (e-mail: edgarcia@unizar.es, cginestra@unizar.es, mariam@unizar.es,_ortigoza@unizar.es,_alfortiz@unizar.es, puimedon@unizar.es, mlsarsa@unizar.es, villar@unizar.es). M. Martínez is temporarily with Università di Roma "La Sapienza”, Dipartimento di Fisica, Piazzale Aldo Moro 5, 00185 Roma (Italy) (e-mail: maria.martinez@roma1.infn.it).

J. Gironnet was with Institut d'Astrophysique Spatiale (IAS). Université Paris-Sud 11, France. He is now with INSU/Division Technique, 1, place Aristide Briand 92195 Meudon Cedex (e-mail: johann.gironnet@dt.insu.cnrs.fr).

T. Rolón is with Universidad Nacional de Asunción, San Lorenzo, Paraguay (e-mail: arolonc@gmail.com). more useful than $(n, \alpha)$ captures in $\mathrm{LiF}$ for spectrometry of fast neutrons.

Index Terms - Bolometers, neutrons, radiation detectors, solid scintillation detectors.

\section{INTRODUCTION}

$I^{N}$ NORGANIC scintillators containing natural Li or enriched in the ${ }^{6} \mathrm{Li}$ isotope have traditionally been used to detect thermal neutrons. The exothermic $(\mathrm{Q}=4.78 \mathrm{MeV})$ capture ${ }^{6} \mathrm{Li}(\mathrm{n}, \alpha){ }^{3} \mathrm{H}$, which has a cross section of 941 barn at $2200 \mathrm{~m} \mathrm{~s}^{-1}$, is the basis for neutron detection. Currently, they are also being taken into consideration for the development of neutron detectors to manage the shortage of ${ }^{3} \mathrm{He}$ [1].

The first proposals of the classic scintillator ${ }^{6} \mathrm{LiI}: \mathrm{Eu}$ to detect slow neutrons [2] and for spectrometry of fast neutrons $[3,4]$ were given in the mid-twentieth century. These papers showed the nonlinearity of its light output with respect to the neutron energy $[3,4]$, and its resolution improvement at low temperatures [5]. Compounds with fluorine, as ${ }^{6} \mathrm{LiF}$ mixed with ZnS:Ag for detection of thermal neutrons [6] or LiF as thermo-luminescent dosimeter [7], were also proposed in the same period. Nowadays, $\mathrm{LiF}$ and ${ }^{6} \mathrm{LiF}$ with different dopants are widely used in both techniques. LiF scintillator has two main drawbacks as a fast neutron spectrometer, even in the favorable case of tungsten doped: low light yield (about 5\% with respect to $\mathrm{NaI}: \mathrm{Tl})$, and long decay time (40 $\mu \mathrm{s})$ of its luminescence $[8,9]$.

Prior works have proposed $\mathrm{LiF}$ bolometers to detect neutrons or even dark matter $[8,10,11,12,13]$. Bolometers profit from the fact that the largest component of the released particles' kinetic energy produces heat and overcomes the low light yield of scintillators. Linearity of the heat signals was verified by irradiations of ${ }^{6} \mathrm{LiF}$ bolometers with monoenergetic neutrons from thermal energy up to $17 \mathrm{MeV}[14,15]$. However, heat signals are much longer (several ms) than light signals and high neutron fluxes cannot be assessed.

The prospects of a cryogenic detector of ${ }^{6} \mathrm{LiF}$ as neutron spectrometer were analyzed by Monte Carlo [16] and they were further designed and built [17]. The unfolding of one spectrum measured with ${ }^{6} \mathrm{LiF}$ was tested later [18].

Sapphire has not traditionally been used for neutron spectrometry though it can be used as dosimeter for high fluxes of neutrons from nuclear reactors [19]. Elastic 
scattering with oxygen or aluminum is the only relevant process for fast neutrons below $1 \mathrm{MeV}$, and accounts for up to $80 \%$ of all interactions in sapphire at $6 \mathrm{MeV}$ [20]. This task is not possible using only the heat signal because heat response is independent of particle type. Hybrid bolometers [21,22,23,24,25] made with scintillating crystals can detect the heat released by the interacting particles in addition to light output. This light output is different for nuclear recoils, alpha particles, electrons and gammas. Hence, a hybrid bolometer of sapphire could be used as a neutron spectrometer based on the scattering by light nuclei because it can distinguish nuclear recoils (produced by neutrons) from electron recoils (produced by gamma rays or electrons) [26]. The technique is also successful for ${ }^{6} \mathrm{LiF}$ or $\mathrm{LiF}$ [27].

This paper reports on the application of two scintillating bolometers of $\mathrm{LiF}$ and sapphire as spectrometers of fast neutrons. The former relies on $(n, \alpha)$ captures and the latter on elastic collisions, which are two typical methods for neutron spectrometry. Preliminary results were reported in $[28,29]$. The work is a byproduct of the ROSEBUD collaboration, focused on the development of scintillating bolometers of different materials for dark matter searches. Due to the relevance of neutron background in such experiments [30], we have used the available data to test the potential of these scintillating bolometers as neutron spectrometers.

\section{SCINTILLATING BOLOMETERS}

The total cross section of $\mathrm{LiF}$ [20] in the region of interest for this work (Fig. 1) is dominated by interactions on ${ }^{19} \mathrm{~F}$ (100\% natural fluorine) and ${ }^{7} \mathrm{Li}$ (92.41\% natural lithium). Elastic scattering is the more probable process for both nuclei. Inelastic scattering accounts for an appreciable fraction of the total cross section only above $0.2 \mathrm{MeV}$ and $0.6 \mathrm{MeV}$ for ${ }^{19} \mathrm{~F}$ and ${ }^{7} \mathrm{Li}$, respectively. Resonances below $0.15 \mathrm{MeV}$ come from pure elastic scattering on ${ }^{19} \mathrm{~F}$ whereas the structure between 0.2 and $0.5 \mathrm{MeV}$ comes from elastic scattering on ${ }^{19} \mathrm{~F}$ plus an important contribution from (n,n') to the first $(110 \mathrm{keV})$ and second (197 keV) excited levels of ${ }^{19} \mathrm{~F}$, as well as a small fraction from resonant elastic scattering on ${ }^{7} \mathrm{Li}$ at $0.24 \mathrm{MeV}$. The cross section of ${ }^{6} \mathrm{Li}$ (Fig. 1 ) is less than $10 \%$ of the total in the region of interest, because of its low natural abundance (7.59\%).

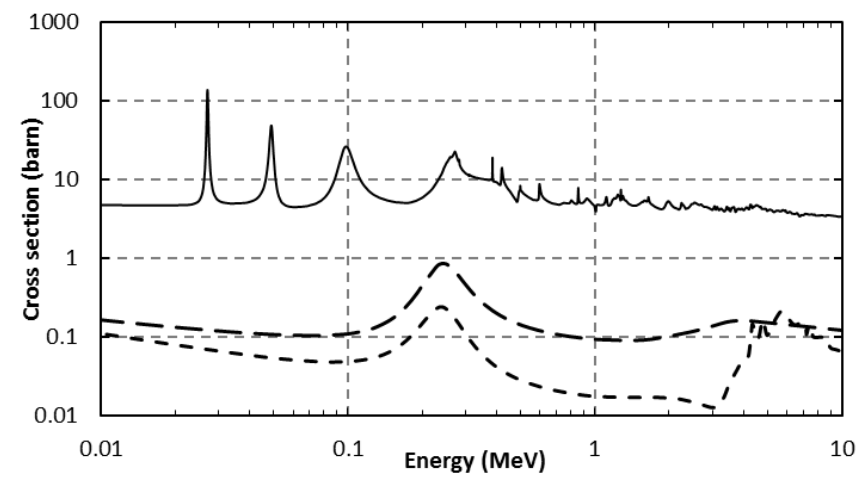

Fig. 1. Total cross section for neutrons on $\mathrm{LiF}$ (continuous line) and ${ }^{6} \mathrm{Li}$ (long dashed line). Short dashed line shows $(n, \alpha)$ cross section on LiF, where capture by ${ }^{6} \mathrm{Li}$ dominates below $3 \mathrm{MeV}$ and capture by ${ }^{19} \mathrm{~F}$ is dominant above $3 \mathrm{MeV}$.

A less common interaction on $\mathrm{LiF}$ is $(\mathrm{n}, \alpha)$ capture (Fig. 1). ${ }^{6} \mathrm{Li}(\mathrm{n}, \alpha)$, with the resonance at $0.24 \mathrm{MeV}$, is dominant for neutron energies below $3 \mathrm{MeV}$, whereas endothermic ${ }^{19} \mathrm{~F}(\mathrm{n}, \alpha)$ $(\mathrm{Q}=-1.52 \mathrm{MeV})$ dominates above $3 \mathrm{MeV}$, and reaches a maximum at $5.7 \mathrm{MeV}$. Note that in a ${ }^{6} \mathrm{LiF}$ bolometer with the abundance of ${ }^{6} \mathrm{Li}$ enriched to $95 \%$, capture by ${ }^{6} \mathrm{Li}$ is 12.5 times higher than that plotted in Fig. 1.

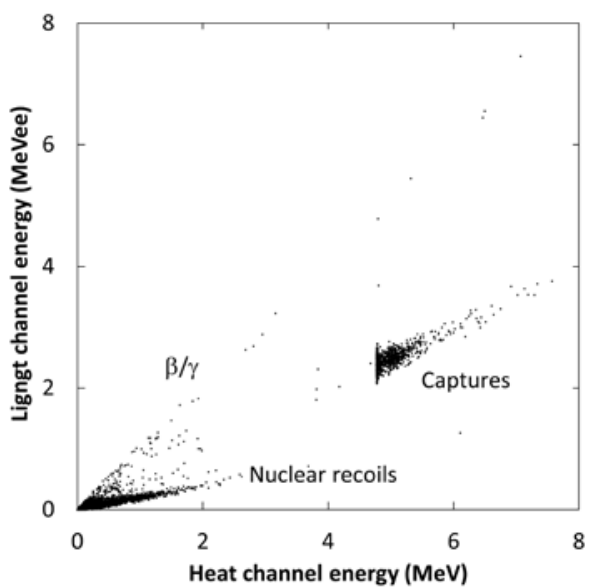

Fig. 2. Light-heat plot of a LiF scintillating bolometer estimated by Monte Carlo with MCNP-PoliMi. Light output is measured in MeVee (MeV electron equivalent) assuming that it is calibrated with gamma rays.

Particle discrimination can be achieved by comparing the light and the heat signals of these particles. Fig. 2 shows an estimate with MCNP-PoliMi v1.0 [31] of a light-heat plot of a $\mathrm{LiF}$ scintillating bolometer irradiated by ${ }^{252} \mathrm{Cf}$. LiF was inside a 25-cm thick lead box in a 1 meter thick rock cavern. This version of MCNP-PoliMi was enough for our purposes; in particular, it considers that charged particles deposit their energy where they are produced, a very good approximation for our problem. To imitate the bolometer response, we assumed similar parameters to those of our LiF scintillating bolometer (see section IV): the heat output does not depend on the type of particle, the light output of the products of neutron captures is 0.5 times the electron light output, the light output of neutron induced nuclear recoils is 0.2 times the electron light output, the resolutions are $1 \%$ in the heat channel and $30 \%$ in the light channel at $4.78 \mathrm{MeV}$ and are extrapolated for all energies according to square root of the energy. If for every history generated by a primary neutron, $E_{r}, E_{e}$ and $E_{c}$ are the transferred energies to LiF by elastic or inelastic neutron collisions, electron absorption and neutron captures, respectively, the expected heat signal, $H_{0}$, is then proportional to $E_{r}+E_{e}+E_{c}$ and the expected light signal, $L_{0}$, is proportional to $0.2 E_{r}+E_{e}+0.5 E_{c}$. Once the pair $\left(H_{0}, L_{0}\right)$ is calculated, every point $(H, L)$ of Fig. 2 is sampled from an uncorrelated two-dimensional Gaussian centered at $\left(H_{0}, L_{0}\right)$ with the standard deviations $\sigma_{h}$ and $\sigma_{l}$ extrapolated from the values at $4.78 \mathrm{MeV}$. Therefore, according to our chosen factors, nuclear recoil events form a high density band 
(nuclear recoils band) with a of slope of 0.2, and interactions of scarce gammas coming from $(n, n$ ') collisions in lead or rock are located on the diagonal $\beta / \gamma$ band. Between the nuclear recoils and $\beta / \gamma$ bands there are (n,n') collisions followed by gamma interaction in $\mathrm{LiF}$. Captures by ${ }^{6} \mathrm{Li}$ of room returned thermal neutrons accumulate at $(4.78 \mathrm{MeV}, 2.39 \mathrm{MeVee})$ whereas $(n, \alpha)$ captures of fast neutrons extend beyond this point as a band of slope slightly lower than 0.5 (captures band) because multiple collisions are possible before a capture event.

Note that in an underground experiment, where the major background source comes from environmental gammas that have energies below $2.6 \mathrm{MeV}$, a hybrid bolometer is not necessary to identify the reaction ${ }^{6} \mathrm{Li}(\mathrm{n}, \alpha)^{3} \mathrm{H}$, because there are no background events of energy greater than $Q$ value. At ground level, however, cosmic ray interactions can transfer energy greater than $\mathrm{Q}$ value. In this case a scintillation signal would be required to detect weak neutron fluxes, given that cosmic ray events are in the $\beta / \gamma$ band and can be easily identified.

The total cross section on sapphire between $10 \mathrm{keV}$ and $10 \mathrm{MeV}$ (Fig. 3) shows that elastic scattering, with oxygen or aluminum, is the only relevant process for fast neutrons below $1 \mathrm{MeV}$. Elastic scattering accounts for up to $80 \%$ of all interactions in sapphire at $6 \mathrm{MeV}$ [20]. Inelastic scattering is the other relevant process and neutron capture is noticeable only above $5 \mathrm{MeV}$. A sapphire bolometer light-heat plot would be similar to Fig. 2 without the capture band.

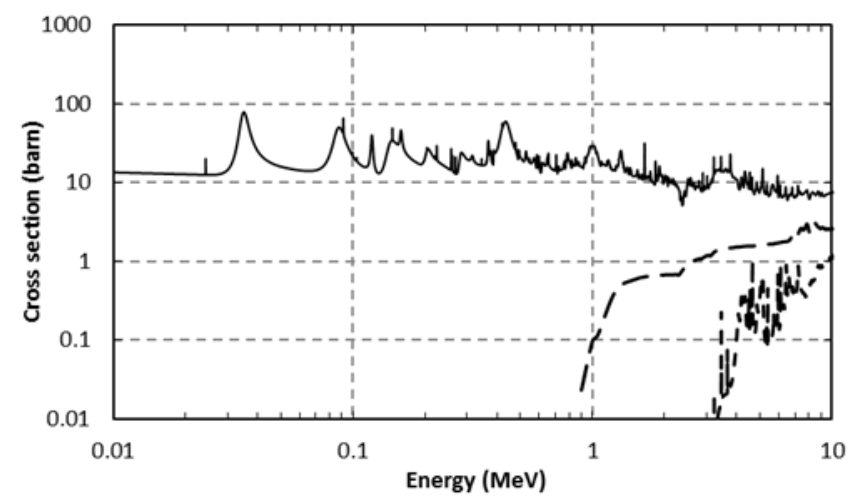

Fig. 3. Total (continuous line), inelastic (long dashed line) and capture (short dashed line) cross sections for neutrons on sapphire.

\section{EXPERIMENTAL SET UP}

Commercial, undoped sapphire and LiF cylindrical crystals (25 mm diameter and $25 \mathrm{~mm}$ height) were used as detector material. Whereas the exact scintillation mechanism is not yet known for the LiF crystal, it is thought to be due to titanium impurities in the sapphire, at the ppm level; see [32] and references therein.

A strong scintillation emission at low temperature was reported for the sapphire crystal, with absolute light yields (fraction of deposited energy going to light) estimated to be 0.127 for gammas, 0.016 for alphas and 0.0073 for neutron recoils, using a strong anticorrelation found on the heat and light signals, and assuming no energy lost in traps [26].
After performing in the double $\mathrm{LiF}$ bolometer a ${ }^{55} \mathrm{Fe}$ calibration of its light detector [33], the collected light yield was estimated to be around $0.4 \mathrm{keV} / \mathrm{MeV}$ for gammas, and $0.11 \mathrm{keV} / \mathrm{MeV}$ for alphas-tritons and $0.06 \mathrm{keV} / \mathrm{MeV}$ for nuclear recoils.

Each crystal (Fig. 4a) was in a copper frame, faced to an optical bolometer, i.e., a thin Ge disk $(25 \mathrm{~mm}$ diameter and several tens of micrometers thickness) absorbing the scintillation light. Bolometers were placed inside a dilution refrigerator at a base temperature of $20 \mathrm{mK}$. Thermistors of Ge-NTD [34] measured the temperature increases of massive and optical bolometers, providing information of the heat and scintillation light produced in the crystals. Signal acquisition was triggered by the logical OR of the signals coming from thermistors, so that both bolometers had the same live time. This was estimated by counting the infrared pulses that, at a frequency of $1 / 30 \mathrm{~s}^{-1}$, were guided via an optical fiber to the bolometers.

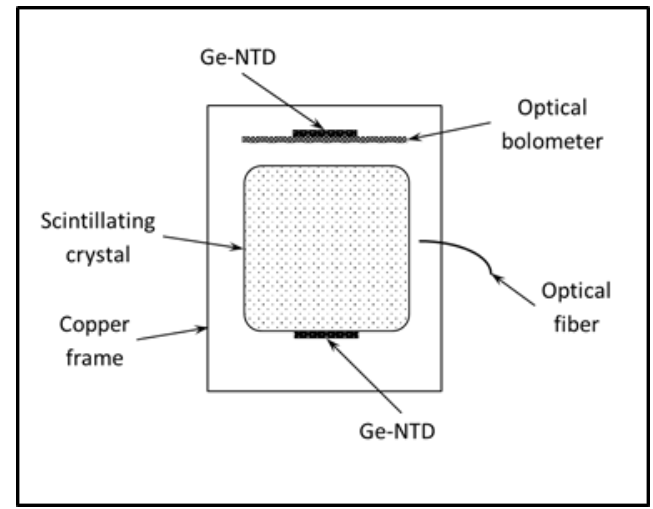

(a)

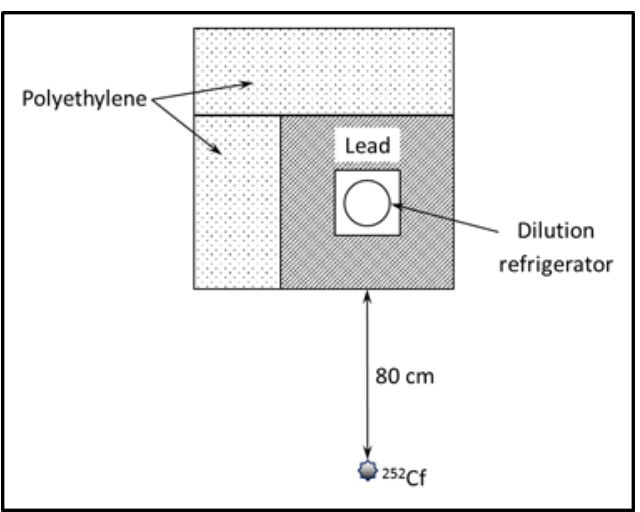

(b)

Fig. 4. (a) Diagram of a scintillating bolometer; (b) Layout of experimental set up (top view)

A source of ${ }^{252} \mathrm{Cf}$ was used to produce the neutron field and to calibrate the $\mathrm{LiF}$ bolometer. The sapphire was calibrated by placing a ${ }^{57} \mathrm{Co}$ source near the bolometers. Underground placement and shielding were required to reduce natural radiation and its associated dead time. The low part of the dilution refrigerator was in the hole of an uncovered lead box of side $80 \mathrm{~cm}$, walls and base of thickness $25 \mathrm{~cm}$. The lead box was on $20 \mathrm{~cm}$ of polyethylene and had two walls covered 
by $40 \mathrm{~cm}$ of polyethylene (Fig. 4b). The system was placed inside a Faraday cage at the formerly called LAB2500 [35], (currently hall E) of the Canfranc Underground Laboratory under $850 \mathrm{~m}$ of rock overburden.

\section{IRRADIATION WITH ${ }^{252}$ CF NEUTRONS}

Both bolometers were irradiated simultaneously for about 8 hours with neutrons emitted by the ${ }^{252} \mathrm{Cf}$ source placed in front of the LiF bolometer, $80 \mathrm{~cm}$ away from the lead wall. The live time was $11430 \mathrm{~s}, 39.2 \%$ of the irradiation time. Dead time is due to the thermal conductance $G$ between the bolometer and the thermistor which, besides the heat capacity $C$ of the bolometer, determines the characteristic time $\tau=C / G$ [14] and the required time to return to the work temperature, $\sim 50 \mathrm{~ms}$ for the bolometers of this work. Note that not only fast neutron interactions contribute to dead time, but also thermal neutron captures and other background events, as $\beta$ and $\gamma$ rays interactions, as well as the pulse processing of the acquisition system. The dead time induced by the absorption of thermal neutrons by $\mathrm{LiF}$ can be suppressed by adding cadmium screens. The dead time induced by $\beta$ and $\gamma$ rays can be reduced using hardware thresholds in the light and/or heat channels to process only the pulses coming from interactions of fast neutrons. Furthermore, the pulses identified as pile-up are rejected because the bolometer temperature after the first event is higher that the work temperature, therefore the increasing of the specific heat gives a nonlinear response for the successive piled-up events. The losses from pile-up could be reduced by adapting new algorithms currently developed for microcalorimeters [36].

The expected neutron spectrum outside the shield is a standard spontaneous fission spectrum, with an additional component due to neutron scattering in the laboratory walls and equipment inside. The source intensity at time of irradiation was $3.72 \times 10^{4}$ neutrons s ${ }^{-1}$, giving a total flux of $0.463 \mathrm{n} \mathrm{cm}^{-2} \mathrm{~s}^{-1}$ at $80 \mathrm{~cm}$ (the nearest lead surface). This value is five orders of magnitude greater than the estimated flux in the LAB2500 [37] or the one measured in "Hall A" [38] of the Canfranc laboratory, both in agreement with typical values of neutron flux underground [39]. Therefore, neutron background is negligible in our data.

\section{A. LiF Data}

The response of the LiF bolometer is plotted in Fig. 5 $[28,29]$ and clearly illustrates the discrimination power of combining light and heat detection.

Events produced by interactions of electrons and photons $(\beta / \gamma$ events) are around a straight line through the origin and the point $(1000,500) \mathrm{mV}$; nuclear recoils are in the band placed mainly below $100 \mathrm{mV}$ in the light signal, and $(\mathrm{n}, \alpha)$ captures are clustered between 2000 and $2100 \mathrm{mV}$ in the heat signal and between 300 and $500 \mathrm{mV}$ in the light signal. Events without a significant heat signal are produced by interactions only in the optical bolometer.

The heat signal spectrum of $(n, \alpha)$ absorptions (Fig. 6) was obtained selecting the events with heat amplitude between
1900 and $2500 \mathrm{mV}$. Absorption of thermal and epithermal neutrons produces the biggest peak around $2000 \mathrm{mV}$ and absorption of fast neutrons peaks around $2100 \mathrm{mV}$. Their energies are $4.78 \mathrm{MeV}$, the $\mathrm{Q}$ value of the ${ }^{6} \mathrm{Li}(\mathrm{n}, \alpha)^{3} \mathrm{H}$ reaction, and $5.02 \mathrm{MeV}$, the $\mathrm{Q}$ value plus $0.24 \mathrm{MeV}$ of the resonance. It was checked that the $5.02 \mathrm{MeV}$ disappears when the neutrons are thermalized [27]. Both peaks give a linear calibration $E(\mathrm{keV})=a+b V(\mathrm{mV})$, where $\quad b=2.636 \pm 0.076 \mathrm{keV} \mathrm{mV}^{-1}$, $a=-499 \pm 152 \mathrm{keV}$, and $\operatorname{cov}(a, b)=-11.485 \mathrm{keV}^{2} \mathrm{mV}^{-1}$. This calibration around $2000 \mathrm{mV}$ cannot be extrapolated to low amplitudes because of big uncertainty (note that an amplitude of $0 \mathrm{mV}$ gives $-499 \pm 152 \mathrm{keV}$ ). The extrapolation has a reasonable precision near $5 \mathrm{MeV}$. For instance, the uncertainty at $3 \mathrm{MeV}$ is about $2 \%$.

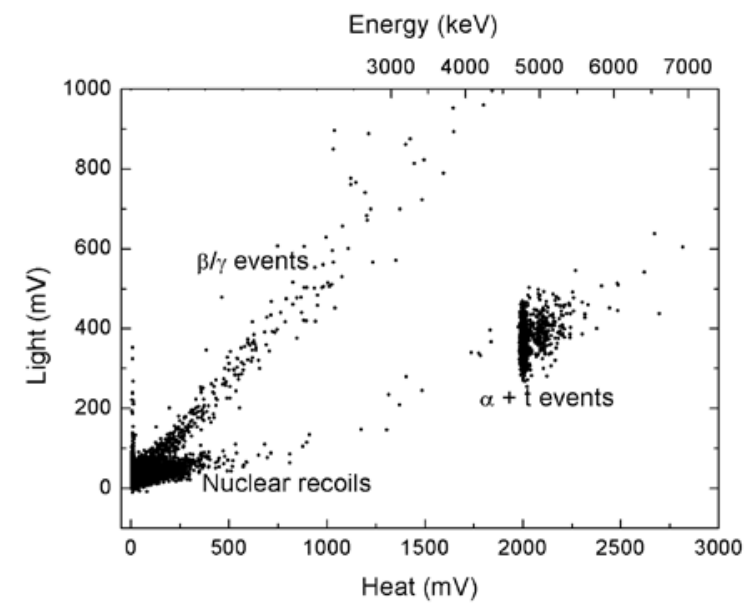

Fig. 5. Light-heat plot of the scintillating bolometer of LiF irradiated with a source of ${ }^{252} \mathrm{Cf}$. Captures ${ }^{6} \mathrm{Li}(\mathrm{n}, \alpha)^{3} \mathrm{H}$ produce $(\alpha+\mathrm{t})$ events, elastic collisions produce nuclear recoils and interactions of electrons and photons produce $(\beta / \gamma)$ events. Events without heat signal are produced by interactions in the optical bolometer. Energy scale (upper axis) is not precise beyond the region around $2000 \mathrm{mV}$, close to ${ }^{6} \mathrm{Li}$ absorption peaks (Fig. 6), due to the extrapolation of the calibration. (C) IOP Publishing. Reproduced with permission. All rights reserved.

Resolution of the heat channel at $4.78 \mathrm{MeV}$ is $54 \mathrm{keV}$; the peak at $5.02 \mathrm{MeV}$ is much wider because it comes from the convolution of the resonance at $0.24 \mathrm{MeV}$ (Fig. 1) and the resolution of heat channel. Figure 5 shows that both peaks are not resolved in the light channel. Then, neutron spectrometry is made with heat signals because its resolution is much better than light signals.

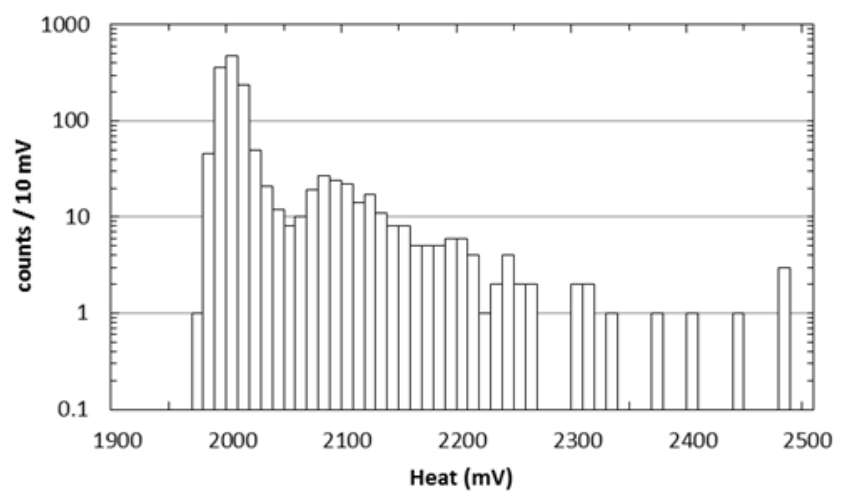

Fig. 6. Spectrum of neutron captures in the scintillating bolometer of $\mathrm{LiF}$ irradiated with a source of ${ }^{252} \mathrm{Cf}$. 
We established a discrimination threshold for the heat signal, where nuclear recoils and $\beta / \gamma$ events are well separated by their light signals. This threshold is approximately at $250 \mathrm{mV}$. Calibration at $250 \mathrm{mV}$ with the ${ }^{57} \mathrm{Co}$ source was not possible since the total absorption peaks of 122 and $136 \mathrm{keV}$ were not visible in the $\beta / \gamma$ band of LiF because they could be below $250 \mathrm{mV}$ or the photoelectric absorption rate of the light $\mathrm{Li}$ and $\mathrm{F}$ atoms could be very weak. Therefore, we did not use the recorded nuclear recoils on LiF for neutron spectrometry.

Energy (keV)

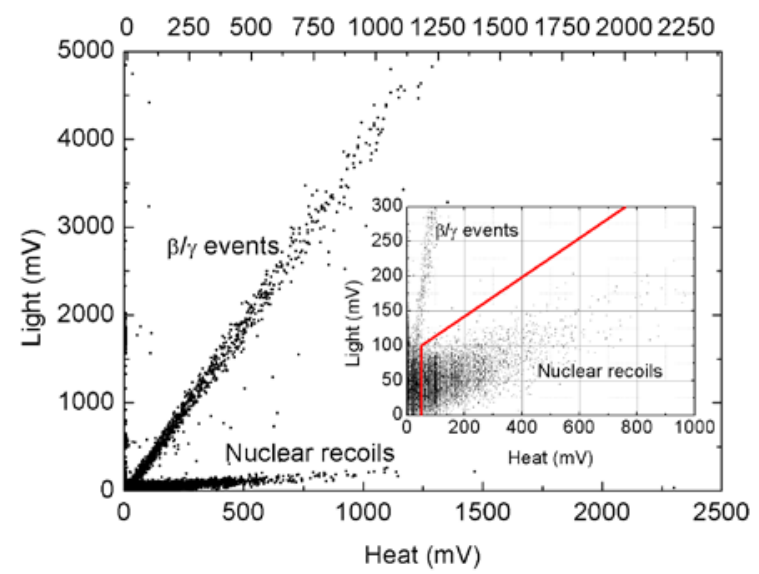

Fig. 7. Light-heat plot of the scintillating bolometer of sapphire irradiated with a source of ${ }^{252} \mathrm{Cf}$. Elastic collisions produce nuclear recoils, and interactions of electrons and photons produce $(\beta / \gamma)$ events. Inset: Detail of the cuts for selecting nuclear recoils. Energy scale (upper axis) is not precise beyond the region around $150 \mathrm{mV}$, close to ${ }^{57} \mathrm{Co}$ energies (Fig. 9), due to the extrapolation of the calibration. (c) IOP Publishing. Reproduced with permission. All rights reserved.

\section{B. Sapphire Data}

The light-heat diagram of sapphire is shown in Fig. 7 $[28,29]$. Nuclear recoils were selected with two cuts: the first one separated events above the discrimination threshold of $50 \mathrm{mV}$ in the heat signal and the second one selected events below the straight line passing through the points $(50,100) \mathrm{mV}$ and $(400,200) \mathrm{mV}$ (see inset, Fig. 7). The spectrum of these events is shown in Fig. 8.

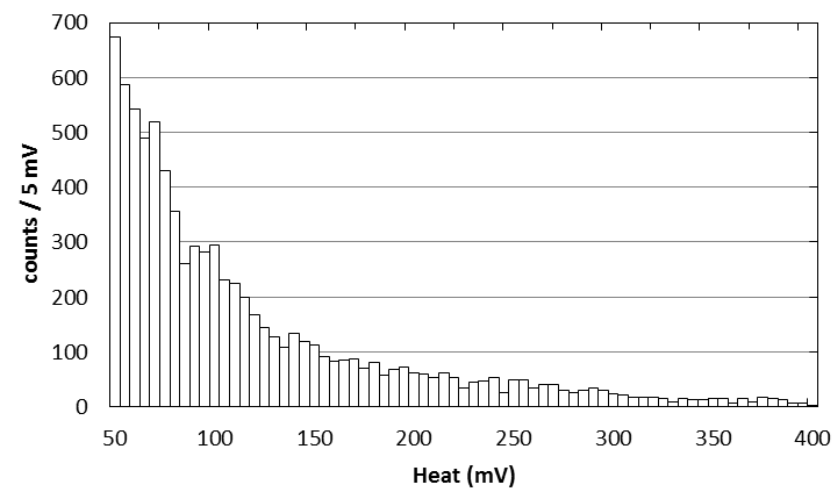

Fig. 8. Spectrum of nuclear recoils in the scintillating bolometer of sapphire irradiated with a source of ${ }^{252} \mathrm{Cf}$.

An estimate of number of nuclear recoils lost in the $\beta / \gamma$ band and of number of $\beta / \gamma$ events included in the nuclear recoil band can be made by fitting events with a heat signal between 50 and $55 \mathrm{mV}$ to two Gaussians in the light signal, one for nuclear recoils and another for $\beta / \gamma$ events. As a result of this fit, we expect only 0.7 lost recoils and only 1.2 included $\beta / \gamma$ events, negligible with respect to the number of events in the bin. The first bin of the recoil spectrum (Fig. 8) remains virtually unaltered by this cut, as does the rest of the bins of Fig. 8 because the discrimination between $\beta / \gamma$ and recoil events is even better than in the bin from 50 to $55 \mathrm{mV}$.

\section{Sapphire Calibration}

The calibration of sapphire with ${ }^{57} \mathrm{Co}$ (Fig. 9) lasted 52 minutes. The $122 \mathrm{keV}$ line is at $139 \mathrm{mV}$ and the $136 \mathrm{keV}$ line appears as an accumulation of about 8 counts at $154 \mathrm{mV}$. To check the position of this line, we used a template obtained in 16.2 hours with ${ }^{57} \mathrm{Co}$ and ${ }^{214} \mathrm{Am}$ (Fig. 10). This calibration had been done in a previous run where an encapsulated source of ${ }^{241} \mathrm{Am}$ was mounted inside the dilution refrigerator, close to the sapphire bolometer [26]. The template was built with a suitable data binning to compare it to Fig. 9.

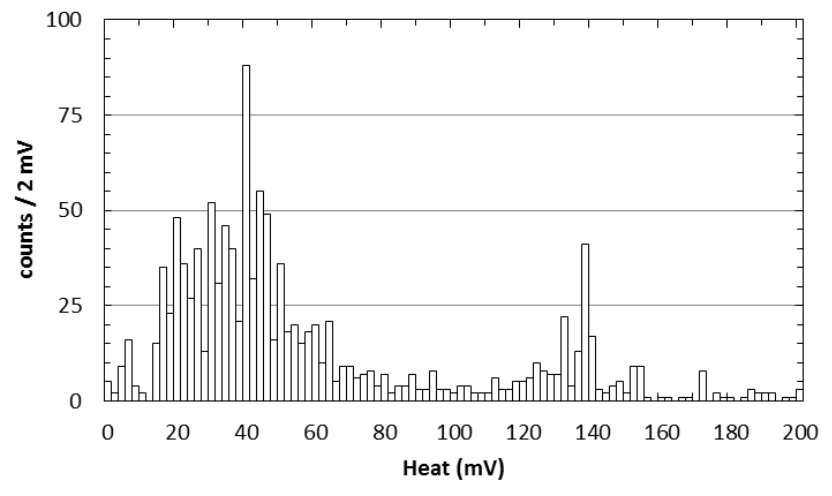

Fig. 9. Calibration spectrum of the scintillating bolometer of sapphire irradiated with a source of ${ }^{57} \mathrm{Co}$.

The lines from ${ }^{57} \mathrm{Co}$ and the line of $59.5 \mathrm{keV}$ from ${ }^{241} \mathrm{Am}$ are clearly visible in Fig. 10, as well as the Compton edge of $122 \mathrm{keV}$ at $39.4 \mathrm{keV}$. The template shows that the accumulation at $154 \mathrm{mV}$ in Fig. 9 matches the $136 \mathrm{keV}$ line and therefore, in spite of low statistics, both ${ }^{57} \mathrm{Co}$ lines can be identified in the calibration. Also, the Compton edge of $122 \mathrm{keV}$ should be locate around $50 \mathrm{mV}$ in Fig. 9.

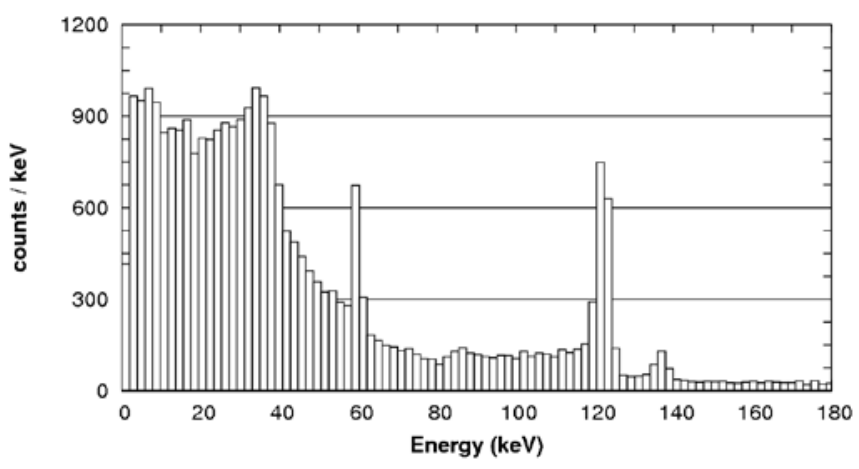

Fig. 10. Template of a calibration spectrum of the scintillating bolometer of sapphire irradiated with ${ }^{241} \mathrm{Am}$ and ${ }^{57} \mathrm{Co}$ [26].

The position of the peaks can be estimated by the maximum likelihood method. Assuming the probability distribution 
function as the normalized addition of two Gaussian functions of the same width plus a second order polynomial as baseline between 100 and $190 \mathrm{mV}$ (Fig. 11), the ${ }^{57} \mathrm{Co}$ lines are located at $139.08_{-0.16}^{+0.15} \mathrm{mV}$ and $154.45_{-1.34}^{+1.14} \mathrm{mV}$ (Table I), with a negligible correlation coefficient of 0.006 . Their estimated areas, $56_{-8}^{+10}$ and $7.0_{-4.0}^{+4.6}$, are compatible with the intensities of $122 \mathrm{keV}$ (85.5\%) and $136 \mathrm{keV}$ (10.7\%) gamma radiations [40].

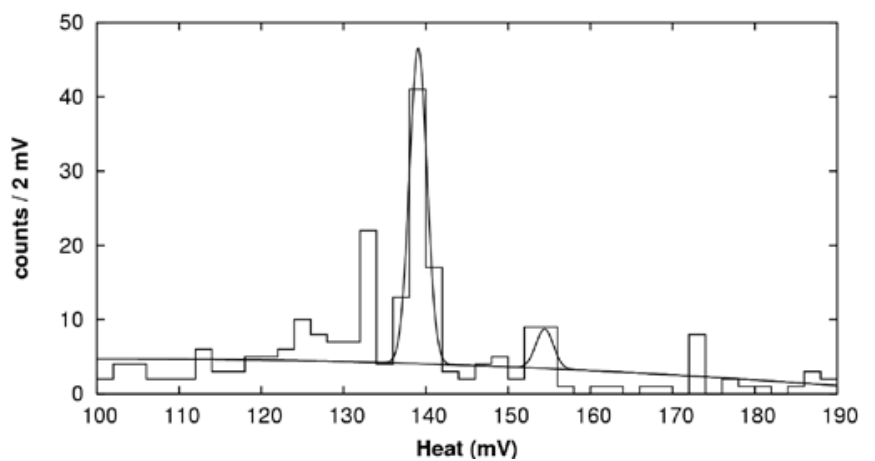

Fig. 11. Plot of the peaks and baseline of the ${ }^{57}$ Co calibration with the estimated parameters by maximum likelihood.

The asymmetric uncertainties have been symmetrized following the method used in "The NUBASE evaluation of nuclear and decay properties" [41]: from the estimated value $X_{-b}^{+a}$, we obtain the symmetric estimate $m \pm \sigma$, where

$$
\begin{aligned}
& \operatorname{erf}\left(\frac{m-X}{\sqrt{2} a}\right)=\frac{a-b}{2 a} \\
& \sigma^{2}=\left(1-\frac{2}{\pi}\right)(a-b)^{2}+a b
\end{aligned}
$$

We take the symmetric estimates in Table I as the calibration points of the sapphire bolometer. According to them, the Compton edge of $122 \mathrm{keV}$ line is located at $52 \pm 7 \mathrm{mV}$, an interval in the right region of Fig. 9. The precision is not good because both peaks are tiny, they are very close together and far from Compton edge.

TABLE I

Estimates OF THE POSITIONS OF ${ }^{57}$ CO PEAKS

\begin{tabular}{ccc}
\hline \hline keV & Maximum likelihood $(\mathrm{mV})$ & Symmetric estimate $(\mathrm{mV})$ \\
\hline 122.06 & $139.08_{-0.16}^{+0.15}$ & $139.07 \pm 0.16$ \\
136.47 & $154.45_{-1.34}^{+1.14}$ & $154.32 \pm 1.24$ \\
\hline \hline
\end{tabular}

\section{Data UnFolding}

Neutron flux is generally a function of position, neutron velocity, and time. In our case, it is time independent and we can suppose the flux is the same in every point of each bolometer surface, since their sizes are much smaller than the relevant lengths. Monte Carlo simulation with MCNP [42] of a simplified geometry (lead, polyethylene and laboratory rock) showed that the flux of the ${ }^{252} \mathrm{Cf}$ fast neutrons inside the hole of the lead shielding where the bolometers are contained is nearly isotropic [43]. Accordingly, flux depends only on the neutron energy.

The spectral flux of the incident neutrons on the bolometers can be estimated using a piecewise constant function:

$$
\emptyset(E) d E=\sum_{k=1}^{N} \emptyset_{k} d E
$$

where the spectral flux of the $k$ th group, $\emptyset_{k}$, is nonzero only inside the interval $\left(E_{k-1}, E_{k}\right)$.

The response $R_{i k}$ is the content of the ith voltage bin per incident neutron of the $k$ th group and was estimated by Monte Carlo with the code MCNP-PoliMi. A simulation including a bolometer, copper frame, helium bath, and lead shielding showed that one emerging neutron from the bolometer had only a $2 \%$ probability of being reflected back inside [43]. This allowed a simplified simulation consisting of a cylinder of same size as the bolometer. We consider negligible the distortion produced by this $2 \%$ fraction in the responses $R_{i k}$.

The experimental spectrum is a linear combination

$$
c_{i} \approx \sum_{k=1}^{N} \alpha_{k} R_{i k}
$$

where $c_{i}$ is the content of the ith bin and the unknown $\alpha_{k}$ is the number of neutrons of the kth group going into the bolometer. Equation (4) is not exact because the model (number and energy intervals of the groups, isotropy of the flux) is approximate. We expect also an inaccuracy of a few percent coming from the simplified geometry used to estimate $R_{i k}$.

Therefore, $\emptyset_{k}$ and $\alpha_{k}$ are related by

$$
\emptyset_{k}=\frac{2 \alpha_{k}}{\Delta S \cdot \Delta t \cdot\left(E_{k}-E_{k-1}\right)}
$$

where $\Delta S$ is the surface of the bolometer, $\Delta t$ is the live time of the measurement and the factor two is due to the assumed isotropy of the neutron flux.

In general, as the number $M$ of bins is greater than the number $N$ of groups, the linear system (4) is over-determined. We chose a model with six groups, of roughly the same width per unit lethargy between 0.05 and $5 \mathrm{MeV}$. It was solved by the method of least squares with the constraint of non-negative flux $\left(\alpha_{k} \geq 0, k=1 \ldots N\right)$ looking for the minimum of

$$
S^{2}=\sum_{i=1}^{M} w_{i}\left(c_{i}-\sum_{k=1}^{N} \alpha_{k} R_{i k}\right)^{2}
$$

The inverse of the estimated variance is the weight of the ith bin

$$
w_{i}=\left(c_{i}+\sum_{k} \alpha_{k}^{2} V_{i k}+\sum_{k, l} \alpha_{k} \alpha_{l} \operatorname{cov}\left(R_{i k}, R_{i l}\right)\right)^{-1}
$$

It assumes that the observed values $c_{i}$ are random variables following the Poisson distribution, it includes the variances [44] $V_{i k}$ of the responses $R_{i k}$ evaluated by MCNP-PoliMi and a third term coming from the uncertainties in the positions of calibration peaks.

The third term takes into account that the output of MCNP-PoliMi has to be binned according to the gain $(\mathrm{keV} / \mathrm{mV})$ and offset of the experimental spectrum. Therefore, this binning depends on the positions $x_{1}$ and $x_{2}$ of the two calibration peaks (see an example in Fig. 12), i.e., $R_{i k}$ is a function $R_{i k}\left(x_{1}, x_{2}\right)$. Then, 


$$
\operatorname{cov}\left(R_{i k}, R_{i l}\right)=\sum_{j, m=1}^{2}\left(\frac{\partial R_{i k}}{\partial x_{j}}\right)\left(\frac{\partial R_{i l}}{\partial x_{m}}\right) \operatorname{cov}\left(x_{j}, x_{m}\right)
$$

Usually, $x_{1}$ and $x_{2}$ are independent or their correlation is negligible, as in our case for the sapphire bolometer:

$$
\begin{gathered}
\operatorname{cov}\left(x_{j}, x_{m}\right)=\sigma_{j}^{2} \delta_{j m},(j=1,2) \\
\operatorname{cov}\left(R_{i k}, R_{i l}\right)=\sum_{j=1}^{2}\left(\frac{\partial R_{i k}}{\partial x_{j}}\right)\left(\frac{\partial R_{i l}}{\partial x_{j}}\right) \sigma_{j}^{2}
\end{gathered}
$$

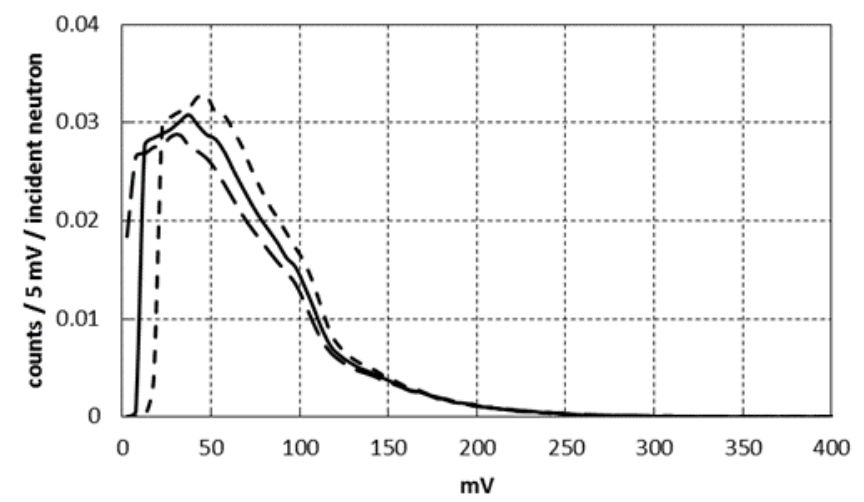

Fig. 12. Response functions of sapphire bolometer for the $3^{\text {rd }}$ group of neutrons, $(0.2,0.5) \mathrm{MeV}$ for different positions of the ${ }^{57} \mathrm{Co}$ peaks. Continuous line $\left(x_{1}=139.07 \mathrm{mV}, x_{2}=154.32 \mathrm{mV}\right)$, short dashed line $\left(x_{1}=140.07 \mathrm{mV}\right.$, $\left.x_{2}=154.32 \mathrm{mV}\right)$, long dashed line $\left(x_{1}=139.07 \mathrm{mV}, x_{2}=155.32 \mathrm{mV}\right)$. Note that, in the sapphire of this work, the useful region is above the $50 \mathrm{mV}$ threshold.

The partial derivatives of (9) were estimated numerically as $\left(R_{i k}\left(x_{j}+\Delta x_{j}\right)-R_{i k}\left(x_{j}\right)\right) / \Delta x_{j}$ for $\Delta x_{j}=1 \mathrm{mV}$. Looking at Fig. 12, they are the difference between the short dashed and the continuous lines $(j=1)$ and the difference between the long dashed and the continuous line $(j=2)$.

TABLE II

RESULtS OF THE UNFOLDING With SiX GROUPS

\begin{tabular}{ccc}
\hline \hline & Sapphire & LiF \\
\cline { 2 - 3 } $\mathrm{MeV}$ & \multicolumn{2}{c}{$\mathrm{s}^{-1} \mathrm{~cm}^{-2} \mathrm{MeV}^{-1}$} \\
\hline $0.05-0.1$ & $<24$ & $<1.7$ \\
$0.1-0.2$ & $0.59_{-0.28}^{+0.39}$ & $0.311 \pm 0.072$ \\
$0.2-0.5$ & $0.248 \pm 0.031$ & $0.315 \pm 0.028$ \\
$0.5-1.0$ & $0.068 \pm 0.012$ & $0.161 \pm 0.040$ \\
$1.0-2.0$ & $0.031 \pm 0.003$ & $<0.28$ \\
$2.0-5.0$ & $0.0017 \pm 0.0007$ & $<1.0$ \\
\hline & \multicolumn{2}{c}{$\mathrm{Flux}\left(\mathrm{s}^{-1} \mathrm{~cm}^{-2}\right)$} \\
& $0.1<\mathrm{E}(\mathrm{MeV})<5$ & $0.1<\mathrm{E}(\mathrm{MeV})<1$ \\
\cline { 2 - 3 } & $0.204_{-0.025}^{+0.036}$ & $0.206 \pm 0.022$ \\
\hline \hline
\end{tabular}

The results of the unfolding for each bolometer are listed in Table II. The first column lists the endpoints of the groups; the second and the third columns are the differential flux estimated with sapphire and $\mathrm{LiF}$ data, respectively. The constrained one-at-a-time uncertainties (or upper limits) of $\emptyset_{k}$ and of the integral flux were estimated at $68 \%$ C. L., as described in [45], Integral fluxes are given in the last row of
Table II. The incident spectral fluxes on each bolometer are plotted in Fig. 13. Solutions give upper limits for the first group between 0.05 and $0.1 \mathrm{MeV}$. This reveals the limited information supported by our data about the flux below $0.1 \mathrm{MeV}$.

The differences between the experimental data and the fitted values, $c_{i}-\sum_{k=1}^{N} \alpha_{k} R_{i k}$, are within their uncertainties. Fig. 14 shows the residuals $w_{i}^{1 / 2}\left(c_{i}-\sum_{k=1}^{N} \alpha_{k} R_{i k}\right)$ at the minimum of (6): differences between data and fitted values of $\mathrm{LiF}$ are within two times the estimated standard deviation in every bin, and those of sapphire are mainly within three times the estimated standard deviation.

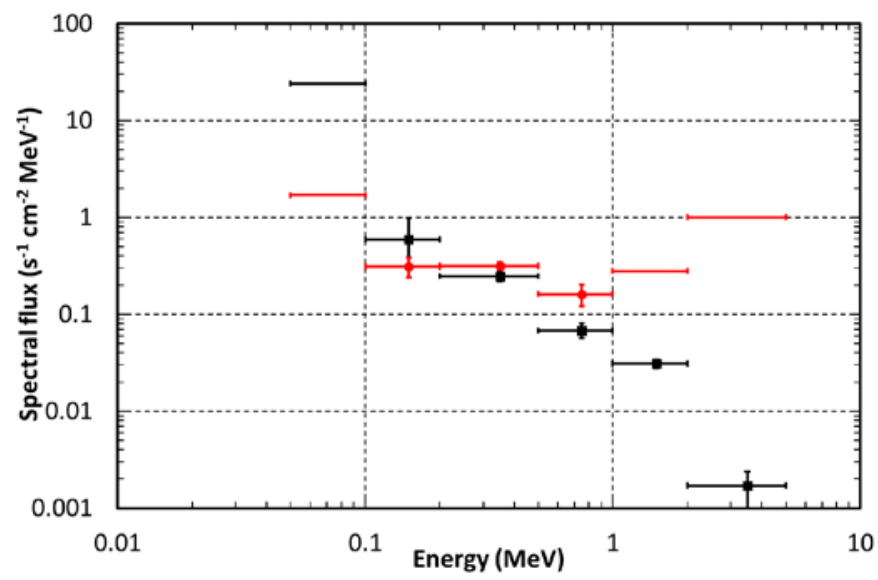

Fig. 13. Results of the unfolding of sapphire data (black squares) and $\mathrm{LiF}$ data (red dots). Only upper limits are plotted for the $1^{\text {st }}$ group, and for $5^{\text {th }}$ and $6^{\text {th }}$ groups for $\mathrm{LiF}$.

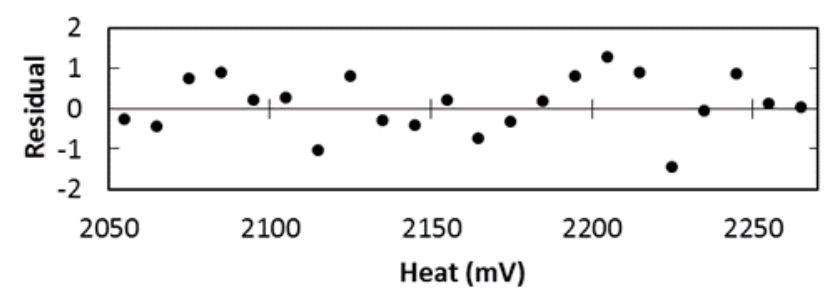

(a)

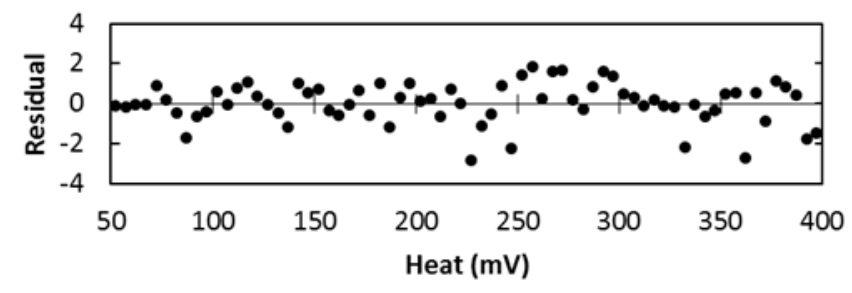

(b)

Fig. 14. Residual plots of the data unfolding of LiF bolometer (a) and sapphire bolometer (b).

No noticeable differences were found taking the weights $w_{i}$ without the variances $V_{i k}$, i.e., the uncertainties of the responses $R_{i k}$ coming from the Monte Carlo method, were negligible in (7). Using one at a time the other summands of the weights $w_{i}$ to minimize (6), we concluded that the randomness of the measured events $c_{i}$ essentially dominates 
the uncertainty of LiF deconvolution and that of sapphire at high energy, whereas the uncertainty at low energy in the sapphire result is dominated mainly by the uncertainty coming from the ${ }^{57} \mathrm{Co}$ calibration. We were not able to improve the resolution of the spectral flux because least squares solutions had strong oscillations when we increased the number of groups, and the unfolding was not possible without an additional constraint of smoothness [46].

\section{CONCLUSION}

The spectral flux of a neutron field has been estimated through scintillation bolometer measurements of the elastic collisions in sapphire and neutron captures in LiF. The live time of the experiment was $39.2 \%$ for $0.2 \mathrm{n} \mathrm{s}^{-1} \mathrm{~cm}^{-2}$. This value is the typical magnitude of the measurable flux of fast neutrons with the set-up here described. In the best conditions, if the processing time was negligible, then the dead time would be $\sim 50 \mathrm{~ms}$ per recorded event, and the saturation count rate would be $\sim 20 \mathrm{~Hz}$ in a nonparalyzable system. Obviously, a realistic upper limit for a measurable rate would be one half of the saturation, say $\sim 10 \mathrm{~Hz}$. As the rate has been $3 \mathrm{~Hz}$ for a flux of $0.2 \mathrm{n} \mathrm{s}^{-1} \mathrm{~cm}^{-2}$, the upper limit for a measurable flux would be $\sim 0.7 \mathrm{n} \mathrm{s}^{-1} \mathrm{~cm}^{-2}$, if the rate was proportional to the neutron flux, i.e., the background was dominated by the $\gamma$ rays and neutrons emitted by ${ }^{252} \mathrm{Cf}$.

Measurements of more intense neutron fluxes could be done with smaller crystals. The chosen mass should depend on the neutron flux and on the background from the rest of sources. This should avoid the increase of the total interaction rate and the corresponding dead time. Furthermore, a smaller mass reduces the heat capacity $C$ and the characteristic time $\tau$. An increasing of the thermal conductance also reduces $\tau$, but it is not recommended because a fraction of the heat could escape of the target before the thermalization were reached, giving a loss in resolution. Bolometers of $25 \mathrm{~g}$ would improve a factor 2 the upper limit of the measurable rate up to $\sim 20 \mathrm{~Hz}$.

Adiabatic Demagnetization Refrigerators (ADRs) could replace dilution refrigerator in the case of ${ }^{3} \mathrm{He}$ shortage.

The LiF bolometer gives good results (within $10 \%$ of uncertainty in the total flux) in the region between 0.1 and $1 \mathrm{MeV}$ even with the small number of events reported in this paper. The peak due to the absorption of thermal neutrons (Fig. 6) masks the capture of fast neutrons below $0.1 \mathrm{MeV}$, while the decreasing of the cross section outside the resonance at $0.24 \mathrm{MeV}$ weakens the sensitivity of $\mathrm{LiF}$ for energies greater than $1 \mathrm{MeV}$ or smaller than $0.1 \mathrm{MeV}$.

The sapphire bolometer gives information above $0.1 \mathrm{MeV}$ and could explore the region below $0.1 \mathrm{MeV}$ with a lower discrimination threshold (Fig. 8). The statistical uncertainty of the unfolded solution for the total flux between 0.1 and $5 \mathrm{MeV}$ is about $15 \%$, which could be improved if the sapphire calibration were more precise. From the point of view of fast neutron spectrometry, detection of elastic collisions by sapphire is a better option than detection of neutron captures by LiF. Note that this is possible because light output classifies nuclear recoils and electron recoils (Fig. 7).
The systematic uncertainty of the simplified Monte Carlo simulation of both bolometers is estimated as $2 \%$, which is the fraction of neutrons that would come back to the bolometer if the materials around were included in the simulated geometry. This uncertainty is negligible with respect to those estimated by the unfolding.

Data unfolding (with a few energy groups) has been obtained without any additional a priori hypotheses other than positive flux because the linear system (4) is over-determined. This is one advantage with respect to a Bonner spheres spectrometer (BSS), where an under-determined linear system has to be solved assuming, a priori, some features of the neutron spectrum. The different available numerical methods to unfold the BSS data give similar total fluxes and spectra of similar shapes but with big discrepancies (a factor two or even a factor ten) for some energy bins; see, for instance, [38]. These disagreements do not exist for scintillating bolometers because the solution of an over-determined system is unique; hence the precision of scintillator bolometers is much better.

Conversely, the method reported in this paper is more complicated and more expensive than a BSS. Nowadays, scintillating bolometers are useful for fluxes no greater than $\sim 1 \mathrm{n} \mathrm{s}^{-1} \mathrm{~cm}^{-2}$, whereas BSS, depending on its features (size, neutron detection based on ${ }^{6} \mathrm{Li},{ }^{3} \mathrm{He}$ or activation foils) can measure neutron fluxes between $\sim 10^{-2}$ and $\sim 10^{4} \mathrm{n} \mathrm{s}^{-1} \mathrm{~cm}^{-2}$ [47].

Due to the limited rate affordable with scintillating bolometers (a few $10 \mathrm{~Hz}$ at most), foreseen applications are restricted for radioprotection topics, such as measurement of the residual fast neutron component after shieldings of the thermal component in accelerators or reactors installations. Other applications are foreseeable with enriched ${ }^{6} \mathrm{LiF}$ detectors, as in situ monitoring of the fast neutron background in experiments looking for dark matter with bolometers, where fast neutrons of energy between $\sim 100 \mathrm{keV}$ and $\sim 1 \mathrm{MeV}$ constitute the ultimate background [48]. They would allow either to check the multi-detector Monte Carlo simulation during the neutron calibration phases, or to monitor the background during the exposure ones. A good deal of ${ }^{6} \mathrm{LiF}$ bolometers could be necessary to compensate for the neutron capture cross section in ${ }^{6} \mathrm{Li}$ with respect to the scattering ones in the targets $\left(\mathrm{Ge}, \mathrm{CaWO}_{4}\right)$ [43] at the relevant neutron energy range.

\section{ACKNOWLEDGMENT}

We thank C. Rolón and C. Wiseman for the proofreading of the manuscript. J. Puimedón thanks B. W. Rust for providing relevant articles on confident intervals for non-negative least squares problems.

\section{REFERENCES}

[1] W. Carel. E. van Eijk, "Inorganic scintillators for thermal neutron detection,” IEEE Trans. Nucl. Sci., vol. 59, no. 5, pp. 2242-2247, Oct. 2012. [2] J. Schenck, “Activation of Lithium Iodide by Europium,” Nature, vol. 171, 518-519, Mar. 1953. 
[3] R. B. Murray, "Use of $\mathrm{Li}^{6} \mathrm{I}(\mathrm{Eu})$ as a scintillation detector and spectrometer for fast neutrons," Nucl. Instrum. Meth., vol. 2, pp. 237-248, Apr. 1958.

[4] D. R. Johnson, J. H. Thorngate and P. T. Perdue, "A sensitive spectrometer for fast neutrons using ${ }^{6} \mathrm{LiI}(\mathrm{Eu})$, , Nucl. Instrum. Meth., vol. 75, pp. 61-65, Nov. 1969.

[5] J. R. P. Eaton and J. Walker, "The response of ${ }^{6} \mathrm{LiI}$ to neutrons as a function of temperature,” Proc. Phys. Soc., vol. 83, pp.301-309, Feb. 1964.

[6] R. Stedman, "Scintillator for thermal neutrons using ${ }^{6} \mathrm{LiF}$ and ZnS(Ag)," Rev. Sci. Instr., vol. 31, p. 1156, Oct. 1960.

[7] J. R. Cameron, F. Daniels, N. Johnson, G. Kenney, "Radiation Dosimeter Utilizing the Thermoluminescence of Lithium Fluoride," Science vol. 134, pp. 333-334, Mar. 1961.

[8] B. Pritychenko et al., "Experimental measurements with a $\mathrm{LiF}(\mathrm{W})$ scintillator,” Nucl. Instrum. Meth., vol. A 396, pp. 371-373, Sep. 1997.

[9] R. Nowotny, "LiF:W as a scintillator for dosimetry in diagnostic radiology,” Phys. Med. Biol., vol. 49, pp. 2599-2611, Jun. 2004.

[10] P. F. Smith et al., "Tests on low temperature calorimetric detectors for dark matter experiments,” Phys. Lett., vol. B 245, pp. 265-270, Aug. 1990.

[11] P.F. Smith et al., "Observation of nuclear recoils events from neutron scattering in low temperature calorimetric detectors,” Phys. Lett., vol. B 255, pp. 454-458, Feb. 1991.

[12] Minowa et al., "Cryogenic thermal detector with LiF absorber for direct dark matter search experiment," Nucl. Instrum. Meth. Vol. A 327, pp. 612-614, Apr. 1993.

[13] P. de Marcillac et al., "Characterization of a $2 \mathrm{~g} \mathrm{LiF}$ bolometer," Nucl. Instrum. Meth., vol. A 337, pp. 95-100, Dec. 1993.

[14] C.S. Silver et al., "Optimization of a ${ }^{6} \mathrm{LiF}$ bolometric neutron detector," Nucl. Instrum. Meth., vol. A 485, pp. 615-623, Jun. 2002.

[15] J. Gironnet, "Spectrométrie de neutrons rapides par bolomètres à cible lithium pour la réduction du fond des expériences de détection directe de matière noire," Ph.D. dissertation, L’Université Paris Sud II U.F.R Scientifique d'Orsay, France, 2010. Available: http://tel.archivesouvertes.fr/tel-00633437.

[16] Z. W. Bell, S. A. Pozzi, E. Padovani, "Monte Carlo analysis of energy deposition in a cryogenic neutron detector," in Nucl. Sci. Symp. Conf. Record, 2005 IEEE (Volume:2), Fajardo (Puerto Rico) pp 1057-1061

[17] I. D. Hau et al., "Neutron absorption spectroscopy for identification of light elements in actinides," Nucl. Instrum. Meth., vol. A 559, pp. 745-747, Apr. 2006.

[18] T. Niedermayr et al., "Unfolding of cryogenic neutron spectra," Nucl. Instrum. Meth., vol. A 579, pp. 165-168, Aug. 2007.

[19] V. I. Flerov, V. Skvortsova and A. V. Flerov, "Thermolumiscence of reactor-irradiated aluminum oxide,” Rad. Prot. Dosim., vol 65, no. 1-4, pp. 227-229, Jun. 1996.

[20] Special Issue on ENDF/B-VII.1 Library, edited by Pavel Obložinský, Nuclear Data Sheets, vol 112, no. 12, Dec. 2011. Available: http://www.nndc.bnl.gov/sigma/index.jsp

[21] L. González-Mestres and D. Perret-Gallix, "Neutrinos, dark matter and low temperature detectors." in Proceedings of the II European Workshop on Low Temperature Detectors for Neutrinos and Dark Matter (LTD-2), Annecy, France, 1988, pp 1-36.

[22] A. Alessandrello et al., "Development of a thermal scintillating detector for double beta decay of ${ }^{48}$ Ca," Nucl. Phys. B (Proc. Suppl.), vol 28, no. 1, pp. 233-235, Jul. 1992.

[23] C. Bobin et al., "Alpha/gamma discrimination with a $\mathrm{CaF}_{2}(\mathrm{Eu})$ target bolometer optically coupled to a composite infrared bolometer," Nucl. Instrum. Meth., vol. A 386, pp. 453-457, Feb. 1997.

[24] P. Meunier et al., "Discrimination between Nuclear Recoils and Electron Recoils by Simultaneous Detection of Phonons and Scintillation Light," Appl. Phys. Lett., vol. 75, no. 9, pp. 1335-1337, Aug. 1999.

[25] N. Coron et al., "Highly sensitive large area bolometers for scintillation studies below 100 mK," Optical Engineering, vol. 43, no. 7, pp.1568-1576, Jul. 2004.

[26] J. Amaré et al., "Light yield of undoped sapphire at low temperature under particle excitation,” Appl. Phys. Lett., vol. 87, art. 264102, Dec. 2005.

[27] J. Gironnet et al., "Neutron spectroscopy with ${ }^{6} \mathrm{LiF}$ bolometers," AIP Conf. Proc., vol. 1185, pp. 751-754, Dec. 2009.

[28] N. Coron et al., "Detection of fast neutrons with $\mathrm{LiF}$ and $\mathrm{Al}_{2} \mathrm{O}_{3}$ scintillating bolometers," J. Phys.: Conf. Ser., vol. 203, pp. 012139.1-012139.2, Feb. 2010.
[29] N. Coron et al., "Measurement of the differential neutron flux inside a lead shielding in a cryogenic experiment," J. Phys.: Conf. Ser., vol. 375, pp. 012018.1-012018.4, Jul. 2012.

[30] L. Baudis, "Direct dark matter detection: The next decade," Dark Universe, vol. 1, pp. 94-108, Nov. 2012.

[31] S. A. Pozzi, E Padovani and M Marseguerra, "MCNP-PoliMi: a MonteCarlo code for correlation measurements," Nucl. Instrum. Meth., vol. A 513, pp. 550-558, Nov. 2003.

[32] Y. Ortigoza et al., "Energy partition in sapphire and BGO scintillating bolometers,” Astroparticle Physics, vol. 34, pp. 603-607, Mar. 2011.

[33] N. Coron et al., "Scintillating and particle discrimination properties of selected crystals for low-temperature bolometers: from LiF to BGO," Nucl. Instrum. Meth., vol. A 520, pp. 159-162, Mar. 2004.

[34] N. Wang et al., "Particle detection with semiconductors thermistors at low temperatures,” IEEE Trans. Nucl. Sci., vol. 36, no. 1, pp. 852-856, Feb. 1989.

[35] I. Bandac et al., "Radón y radiación ambiental en el Laboratorio Subterráneo de Canfranc (LSC),” Radioprotección, vol. XXI, no. 37, pp. 36-41, Jan. 2014.

[36] J.W. Fowler et al., "Microcalorimeter Spectroscopy at High Pulse Rates: a Multi-Pulse Fitting Technique," The Astrophysical Journal Supplement Series, vol. 219, no. 2, art. no. 35, Aug. 2015.

[37] J. M. Carmona et al., "Neutron background at the Canfranc underground laboratory and its contribution to the IGEX-DM dark matter experiment," Astroparticle Physics, vol. 21, pp. 523-533, Aug. 2004.

[38] D. Jordan et al., "Measurement of the neutron background at the Canfranc Underground Laboratory LSC", Astroparticle Physics, vol. 42, pp. 1-6, Feb. 2013.

[39] G. Heusser, "Low-Radioactivity Background Techniques", Ann. Rev. Nucl. and Part. Sci. vol. 45, pp. 543-590, Dec. 1995.

[40] E. Browne and R. B. Firestone, Table of radioactive isotopes. V. S. Shirley Ed. New York: John Wiley \& Sons, 1986, pp. 57-2.

[41] G. Audi, O. Bersillon, J. Blachot and A.H. Wapstra, "The NUBASE evaluation of nuclear and decay properties," Nucl. Phys. A, vol. 72, pp. 3-128, Dec. 2003.

[42] J. F. Briesmeister (ed), "MCNP - A general Monte Carlo N-Particle transport code, version 4C," LA-13709-M, 2000.

[43] T. A. Rolón, "Detección de neutrones con bolómetros centellantes de fluoruro de litio y zafiro y su aplicación en experimentos de búsqueda de materia oscura,” Ph.D dissertation. Dept. Física Teórica, Zaragoza Univ., Zaragoza, Spain. 2013. Available:

http://www.unizar.es/lfnae/rosebud/PhD_Thesis/Tesis_TomasRolon.pdf [44] J. Pulpán and M. Králík, "The unfolding of neutron spectra based on the singular value decomposition of the response matrix," Nucl. Instrum. Meth., vol. A 325, pp. 314-318, Feb. 1993.

[45] B. W. Rust and D. P. O' Leary, "Confidence Intervals for Discrete Approximations to Ill-Posed Problems,” J. Comp. Graph. Stat., vol. 3, pp. 67-96, Mar. 1994.

[46] M. Reginatto, "Overview of spectral unfolding techniques and uncertainty estimation,” Radiation Measurements, vol. 45, pp. 1323-1329, Dec. 2010.

[47] D. J. Thomas and A. V. Alevra, "Bonner sphere spectrometers - a critical review,” Nucl. Instrum. Meth., vol. A 476, pp. 12-20, Jan. 2002.

[48] G. Angloher et al., "EURECA Conceptual Design Report," Physics of the Dark Universe, vol. 3, pp. 41-74, Apr. 2014. 